\title{
Short term outcome in severe alcoholic hepatitis patients treated with Methylprednisolone plus $\mathbf{N}$ acetylcysteine or Pentoxifylline plus $\mathrm{N}$ acetylcysteine.
}

\author{
Kiran Regmi ${ }^{1}$, Anil Kumar Mishra ${ }^{2}$, Sudhamshu KC ${ }^{2}$, Dilip Sharma ${ }^{2}$, Jeetendra Kaji Shrestha ${ }^{3}$, Sushil \\ Prajapati $^{3}$, Dipendra Khadka ${ }^{4}$
}

${ }^{1}$ Consultant Physician, Department of Medicine, Pokhara Academy of Health Sciences, Pokhara, Nepal

${ }^{2}$ Professor, Liver Unit Bir Hospital, Kathmandu, Nepal

${ }^{3}$ Asst. Professor, Liver Unit Bir Hospital, Kathmandu, Nepal

${ }^{4}$ Consultant Physician, Department of Medicine, Nepalgunj Medical Collage, Nepalgunj, Nepal

\section{Correspodence :}

Dr. Kiran Regmi, Consultant Physician, Department of Medicine,

Pokhara Academy of Health sciences,

Pokhara, Nepal

email: regmikeeran@gmail.com

Article recived : 17th July 2018

Article accepted : 1st Oct. 2018

\begin{abstract}
Introduction: Severe Alcoholic hepatitis (AH) is an acute form of alcohol induced liver injury. Often it present as fetal diseases with very high $(30-50 \%)$ short term (28 days) mortality. This study was conducted from period May 2016 to July 2017 in Liver unit, Bir hospital. The main objective was to find out 28 days mortality in patients with severe alcoholic hepatitis who had Discriminant function (DF) $\geq 32$. This was a prospective, comparative, randomized interventional hospital based study.

Methodology: Hundred and ten diagnosed patients of severe alcoholic hepatitis who fulfilled the criteria were enrolled and randomized into two groups (odd number and even number). Group 1 received methylprednisolone and group 2 received pentoxifylline for 28 days. In both groups $\mathrm{N}$ acetylcysteine were added. Lille score was calculated in methylprednisolone group at day 7 and patients with score of $\leq 0.45$ were continued methylprednisolone for total 28 days otherwise stopped. Data were recollected at day 28 . They were compared in relation to survival, complications of drugs and causes of mortality.

Results: Mean age of presentation were $40.21 \pm 10.5 \mathrm{yrs}$ in methylprednisolone and $42.1 \pm 12.1 \mathrm{yrs}$ in pentoxifylline group. In both groups complications were nausea, vomiting, bloating, anorexia and swelling of limb. However, hyperglycemia (16.4\%) and renal impairment $(9.1 \%)$ were more common in methylprednisolone group. Mortality rates were $34.5 \%$ in methylprednisolone and $37.8 \%$ in pentoxifylline group within 28 days. Common causes of death in both groups were hepatic encephalopathy, hepatorenal syndrome, sepsis or the cause was undetermined.

Conclusion: Alcoholic hepatitis is common manifestation of alcoholic liver disease with high short term mortality in both the groups however adverse effects of drugs are more common in methylprednisolone groups.

Keywords: Alcoholic hepatitis, methylprednisolone, pentoxifylline, discriminant function, model for end stage liver diseases.
\end{abstract}

\section{INTRODUCTION}

$\mathrm{AH}$ is a clinical entity with rapid onset of jaundice with elevated serum aspartate transaminase (AST), arising on the background of heavy alcohol use. The thresholds for amount and duration of alcohol use causing $\mathrm{AH}$ are not known, although an average consumption of more than 3 drinks $(\sim 40 \mathrm{~g})$ per day for women and 4 drinks ( $50-60 \mathrm{~g})$ per day for men are reasonable minimal thresholds for the diagnosis of AH. Patients typically have history of heavy drinking for $>5$ years, but may be intermittently abstinent. For the diagnosis of $\mathrm{AH}$, heavy alcohol use should have occurred for $>6$ months, with $<60$ days of abstinence before the onset of jaundice. ${ }^{1}$

Heavy drinkers are at risk for a spectrum of histologic alcohol-related liver injury: steatosis, alcoholic 
steatohepatitis (ASH), alcohol-related fibrosis, and cirrhosis. Alcoholic hepatitis $(\mathrm{AH})$, the clinical entity associated with severe $\mathrm{ASH}$, has high short-term mortality. ${ }^{1}$ In a study short term mortality (30 days) in severe alcoholic hepatitis was $12-15 \%{ }^{2}$ or high as $21 \%{ }^{3}$ Jaundice is often accompanied by malaise, tender hepatomegaly, and decompensation (ascites, encephalopathy, bacterial infection, and variceal bleeding). Serum bilirubin is usually elevated $(>3 \mathrm{mg} / \mathrm{dL}$ ), as is AST (>50 IU/L), and AST to alanine aminotransferase (ALT) ratio of $>1.5$. The AST and ALT do not typically exceed $400 \mathrm{lU} / \mathrm{mL}$, distinguishing $\mathrm{AH}$ from other liver diseases such as drug-induced liver injury (DILI) and ischemic hepatitis. ${ }^{1}$

As alcohol related disorders are common in Nepalese population and there are no study regarding severe $\mathrm{AH}$. This study aimed to find out the short term (28 days) mortality in severe $\mathrm{AH}$.

\section{MATERIALS AND METHODS}

This is a prospective, randomized and interventional study conducted in the Liver unit of Bir hospital, Nepal. Consecutive patients of alcoholic hepatitis who were attended Out Patients Department (OPD) or In Patients Department (IPD) of Liver unit or Emergency department between periods of May 2106 to July 2017 was enrolled. Study was approved by Institutional Review Board (IRB) of NAMS, Bir hospital. The required sample size was calculated on the assumption that the survival rate would be $67 \%$ at 6 months in the methyl prednisolone-only group. With an alpha error of 0.05 , a beta error of 0.20 , and a hypothetical improvement in survival of $20 \%$ at month 6 for the methyl prednisolone plus $\mathrm{N}$-acetylcysteine group ${ }^{4}$ the required sample size was 108 patients. Inclusion criteria were any patients who had ongoing alcohol drinking or consumed during last 2 months period with discriminant function (DF) $\geq$ 32 and AST IALT ratio more than 2. Exclusion criteria include age less than 18 years, pregnancy, viral hepatitis, hepatocellular carcinoma, patients who did not gave consent and patients who were also enrolled in other clinical trials at the same period.

Detailed history, examinations (general and systemic) with recording of vital signs, abdominal girth were taken. Relevant baseline investigations like complete blood counts, Liver function tests, Prothombin Time/ International normalization ratio, renal function tests and random blood glucose were sent. Other tests like Hepatitis B surface antigen, Anti Hepatitis $C$ virus antibody and Human immunodeficiency virus (rapid kit test) were also sent. B mode ultrasound of abdomen and Doppler Ultrasound abdomen were done in all cases to look for absence/presence of liver cirrhosis and hepatocellular carcinoma. Upper Gastrointestinal endoscopy was carried out in all patients before starting methylprednisolone or pentoxifylline. Special tests like antinuclear antibody, anti smooth muscle antibody, liver kindey microsomal antibody, anti mitochondrial antibody, PANCA along with iron profile, ceruloplasmin, were sent in selected patient when required to rule out other causes of hepatitis. CAGE questionnaire was complied in all patients with score of $\geq 2$ taken as significant alcohol abusers or dependent requiring intervention.

CAGE questionnaire comprise of four questions:

1. Have you ever felt you needed to Cut down on your drinking?

2. Have people Annoyed you by criticizing your drinking?

3. Have you ever felt Guilty about drinking?

4. Have you ever felt you needed a drink first thing in the morning (Eye-opener) to steady your nerves or to get rid of a hangover?

After detail examinations and admission these patients DF and ratio of AST to ALT were calculated. Patients were considered for therapy if DF $\geq 32$ and AST/ $A L T \geq 2$ and if there were no contraindications. Daily, detailed examinations and routine blood tests along with necessary other tests were carried out. Clinical measures observed were daily weight, abdominal girth, urine output, features of hepatic encephalopathy sign of sepsis, bleeding or rashes.

Those patients were divided into two groups for randomization, odd number got methylprednisolone plus $\mathrm{N}$ acetylcysteine (Group 1) and even number got pentoxifylline plus $\mathrm{N}$ acetylcysteine (Group 2). In group 1, patients were given oral methylprednisolone $32 \mathrm{mg}$ daily for 4 weeks plus $\mathrm{N}$ acetylcysteine 140 $\mathrm{mg} / \mathrm{kg}$ loading dosages, followed by $70 \mathrm{mg} / \mathrm{kg}$ every 4 hourly for next 17 dosages. Lille score was carried out at day 7 following treatment in the first group and if it was $\leq 0.45$ treatment was continued for 28 days then tapered and stopped (16 mg methylprednisolone for 3 days followed by $8 \mathrm{mg}$ daily for 3 days then $4 \mathrm{mg}$ daily for 3 days and then $2 \mathrm{mg}$ daily for 3 days before stopping). Group 2 were given oral pentoxifylline 400 $\mathrm{mg}$ three times daily plus oral $\mathrm{N}$ acetylcysteine (140 $\mathrm{mg} / \mathrm{kg}$ loading dosage, then $70 \mathrm{mg} / \mathrm{kg}$ every 4 hourly 
for next 17 dosages). These patients (in both groups) stayed in hospital for minimum 5 days. Following discharge they were reviewed at end of first week and at 28 days. However they were followed up whenever felt necessary by patients or by attendant. Data were taken at the end of the first week and at first month. Different scoring like discriminant function (DF), Albumin Billirubin INR Creatinine (ABIC), Glasgow alcoholic hepatitis score (GAHS) Model for end stage liver disease (MELD), Child Turchotte Pugh (CTP) score) were calculated by different formulas as they are mathematical model. ${ }^{5}$ During follow up detailed clinical examination were done together with routine blood tests and recording of any complications. They were also asked for compliance to drugs, nutrition, and abstinence of alcohol.

Data were entered by using Microsoft excel 2007. Statistical analysis was done using SPSS (version 23) software. Quantitative variable expressed as means $\pm S D$ and compared with the use of the Wilcoxon test, Kruskal-Wallis tests or Student $t$ tests as appropriate. Qualitative variables were expressed as percentage, and compared with chi squared test. Kaplan-Meier survival curves was plotted at the end of 28 days and compared with use of log rank test. A 95\% confidence interval was taken and $P$ value less than 0.05 were termed as statistically significant.

\section{RESULTS}

During study period 116 patients fulfilled the inclusion criteria. 6 patients were excluded (two patients with chronic hepatitis $B$ viral infection, two patients with anti nuclear antibody positive, two patients had active peptic ulcer diseases). Finally, 110 patients were available for the study. 55 patients each were randomized into 2 groups. Male patients were predominant in both groups. Minimum age was 25 years and maximum age was 78 years.

Most common age group at presentation was between 31 to 40 years $n=35(31.5 \%)$ followed by 41 to 50 years $n=30(27.27 \%)$. Similarly, 20 to 30 years $n=23(20.90 \%)$, age between 51 to 60 years $n=16(14.54 \%)$, age between 61 to 70 years $n=5(4.5 \%)$ and age between 71 to 80 years $n=1(0.9 \%)$.

At presentation, various clinical features included jaundice, abdominal distension, altered sensorium, fever, abdominal pain as shown in figure 1. Similarly on abdominal examination abdominal distension was most common $(72.7 \%)$ in both groups and other findings were as shown in table 1.

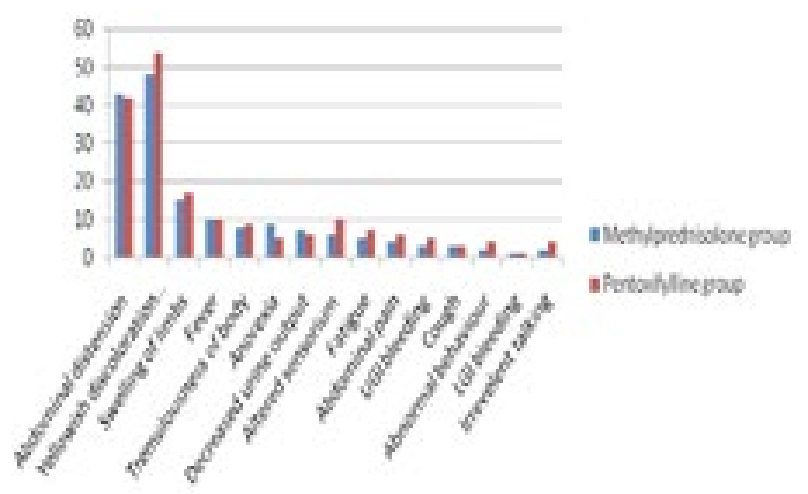

Fig 1: Clinical features

Table 1: Findings of Abdominal examination

Abdominal Methylprednisolone Pentoxifylline Chi P examination group group square value

Abdominal

$\begin{array}{lllll}\text { distension } & 40(72.7) & 40(72.7) & 0 & 1\end{array}$

$\begin{array}{lllll}\text { Liver tenderness } \quad 14(25.5) & 9(16.4) & 1.374 & 0.241\end{array}$

Mean liver

size $(\mathrm{cm}) \quad 15.5 \pm 2.0(11-20) \quad 15.5 \pm 1.9 \quad-0.647 \quad 0.519$

$\begin{array}{lllll} & & (12-20) & & \\ \text { Hepatomegaly } & 29(52.72) & 31(56.36) & 0.147 & 0.702\end{array}$

$\begin{array}{lllll}\text { Palpable spleen } & 15(27.3) & 22(40) & 1.96 & 1.58\end{array}$

$\begin{array}{lllll}\text { Ascites } & 33(60) & 40(72.2) & 1.99 & 0.158\end{array}$

$\begin{array}{lllll}\text { Hepatic bruit } & 3(5.5) & 4(7.3) & 0.806 & 0.369\end{array}$

At admission these patients were evaluated and different scoring (Discriminant function, Lille score, AIBC score, MELD score, GASH score and CTP score) were calculated and similar calculation were also carried out on day 28. The results of different scores are as depicted on table 2 and 3.

Table 2: Different scoring on admission.

\begin{tabular}{|c|c|c|c|c|}
\hline $\begin{array}{l}\text { Scoring } \\
\text { (Mean) }\end{array}$ & $\begin{array}{l}\text { Methylprednisolone } \\
\text { group }\end{array}$ & $\begin{array}{l}\text { Pentoxifylline } \\
\text { group }\end{array}$ & T test & $\begin{array}{c}P \\
\text { value }\end{array}$ \\
\hline DF & $61 \pm 24(33-129)$ & $59.8 \pm 35(32-214)$ & 0.383 & .702 \\
\hline AIBC & $6.9 \pm 1.2(4.5-10)$ & $7.17 \pm 1.2(4.9-10.27)$ & -0.736 & 0.463 \\
\hline MELD & $21 \pm 4.9(10-29)$ & $21.2 \pm 5.7(11.2-40)$ & 0.013 & 0.990 \\
\hline GAHS & $8.54 \pm 0.6(7-10)$ & $8.5 \pm 0.6(7-10)$ & 0.144 & 0.886 \\
\hline CTP & $10.5 \pm 0.9(8-13)$ & $10.5 \pm 1.0(7-12)$ & -0.193 & 0.847 \\
\hline
\end{tabular}

Table 3: Different scoring in Day 28.

\begin{tabular}{llllc}
$\begin{array}{l}\text { Scoring } \\
\text { (Mean) }\end{array}$ & $\begin{array}{c}\text { Methylprednisolone } \\
\text { group }\end{array}$ & $\begin{array}{c}\text { Pentoxifylline } \\
\text { group }\end{array}$ & T test & \multicolumn{1}{c}{$\begin{array}{c}\text { galue } \\
\text { value }\end{array}$} \\
DF & $34.8 \pm 19.7(5.8-124)$ & $40.6 \pm 21(13-107)$ & -1.294 & 0.200 \\
ABIC & $6.2 \pm 1.1(3.9-8.7)$ & $6.2 \pm 1.3(2.15-8.9)$ & -0.110 & 0.913 \\
MELD & $17.7 \pm 4.7(8-33)$ & $18.9 \pm 5.5(7.17-40)$ & -0.965 & 0.338 \\
GAHS & $8.0 \pm 0.6(7-9)$ & $7.9 \pm 0.7(7-9)$ & 0.850 & 0.398 \\
CTP & $9.7 \pm 1.1(8-12)$ & $10.2 \pm 1.2(7-12)$ & -1.795 & 0.077
\end{tabular}

Several complications were noted in both groups 
during study period. Among them most common are shown in fig 2

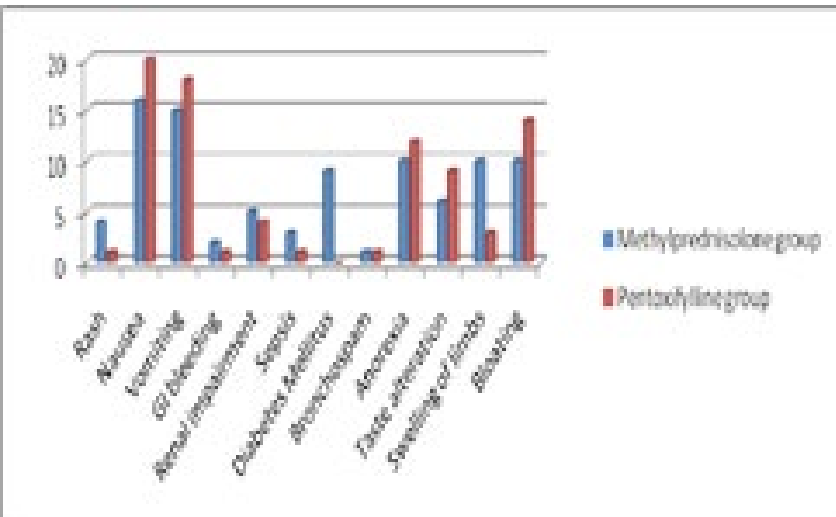

Fig: 2 Complications of drugs

Mortality in methylprednisolone group was $34.5 \%$ and pentoxifylline group $37.8 \%$ which was not statistically significant $\quad(P=0.692)$. In methylprednisolone group commonest cause of death was hepatic encephalopathy $n=6(10.9 \%)$ followed by hepatorenal syndrome $n=3(5.5 \%)$ and sepsis $n=3(5.5 \%)$. Similarly in pentoxifylline group commonest cause of death was hepatic encephalopathy $n=5(9.1 \%)$ and undetermined $\mathrm{n}=5(9.1 \%)$ followed by $\mathrm{GI}$ bleeding $\mathrm{n}=4(7.3 \%)$. In CTP grading most of the patients in both groups were CTP C (87.27\%) and CTP B. Regarding survival there were no statistically significant difference between both groups which is shown in fig. 3

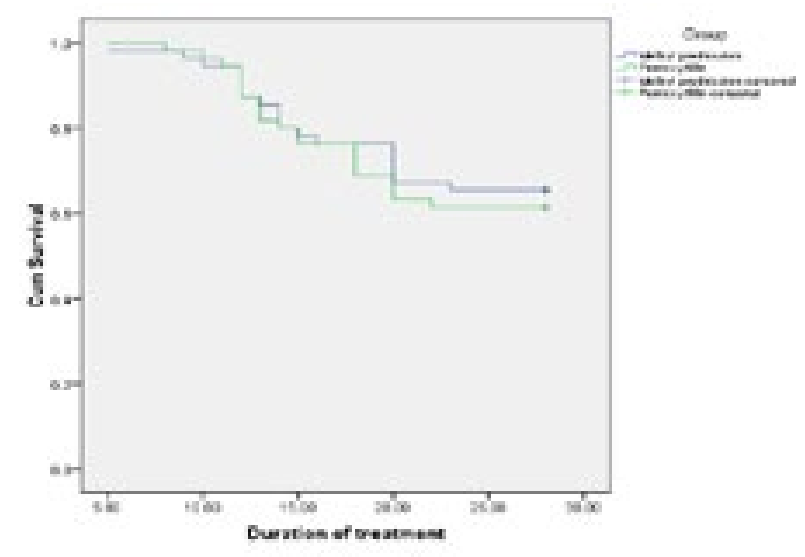

Fig 3: Survival among groups

\section{DISCUSSION}

Alcoholic hepatitis is clinical entity which is further divided into mild form and severe form. Severe form had discriminant function $\geq 32$ or MELD score $>21$ or GAHS $>9$. Mild AH have $90 \%$ survival within 90 days $^{6}$ with supportive care like abstinence of alcohol, good nutrition (30-40 kcal/day), multivitamins and management of alcohol dependence with benzodiazepines. Nutritional support improves liver function and short-term follow-up studies suggest that improved nutrition might improve survival times and histological findings in patients with $\mathrm{AH}^{7} \quad \mathrm{~A}$ large multicentre randomized (STOPAH) trial was conducted in the United Kingdom between 2011 and 2014, in patients with clinical diagnosis of severe $\mathrm{AH}$. There was controversy regarding use of corticosteroids or pentoxifylline $(\mathrm{PTX}) .^{8}$ This study reported a borderline reduction in mortality at 28 days for patients treated with prednisolone $40 \mathrm{mg} /$ day compared with control patients. In a meta analysis, after one month prednisolone therapy provided no benefit in patients of alcoholic hepatitis. ${ }^{9}$ In AAC- NAC4 trial where 174 patients of severe $\mathrm{AH}$ were included and divided into two groups and treated with prednisolone only or with NAC plus prednisolone, 6 month mortality was $38 \%$ in prednisolone group and $27 \%$ in NAC plus prednisolone group, however it was not statistically significant $(P=0.07)$. Hepatorenal syndrome were observed less in NAC plus prednisolone group compared to prednisolone group only $(P=0.02)$. Similarly 1 month mortality was lower in combination group than in prednisolone group $(\mathrm{P}=0.006)$.

In other studies Mathurin and colleagues ${ }^{10}$ observed higher mortality in patients who were not treated with steroid than treated with steroid $(34.3 \%$ vs. $20.03 \%)$ within 28 days period.

However in our study mortality rate was quite high $34.5 \%$ in methylprednisolone group and in $37.8 \%$ in pentoxifylline group in 28 days period. Most clinical trials for $\mathrm{AH}$ have used this score based on its use in the original corticosteroid trials. A number of other scoring systems have also been validated and generally performed similar to the Maddrey score, including the MELD score, Age Bilirubin INR Creatinine (ABIC) score, and the Glasgow scale ${ }^{11}$ A MELD score $>20$ has been proposed as defining severe $\mathrm{AH}$ with an $\sim 20 \%$ mortality. ${ }^{12}$ Lille score (a continuous score with a scale from 0 to 1 ) at $4-7$ days of corticosteroids therapy can be used to assess the response to corticosteroids. Lille score $\leq 0.45^{13}$ combined score has been compared to the CTP score, MELD, and MDF, and shown to have an overall sensitivity of $73 \%$ and specificity of $83 \%$, which was at least as good as other scoring systems. ${ }^{14}$ In a study by Carinthers ${ }^{15}$ 
Orginal Article Medical Journal of Pokhara Academy of Health Sciences (MJPAHS) Vol. 1 Issue 2 Jul-Dec 2018

and olleagues where 66 patients were enrolled with severe alcoholic hepatitis and randomized to take placebo or methylprednisolone $32 \mathrm{mg}$ for 4 weeks followed by tapering in next 2 week. Major outcome were in placebo group $(n=31)$ where $11(35 \%)$ patients died in 28 days while in methylprednisolone group $(n=35)$ where $2(6 \%)$ patients died in same period $(\mathrm{P}=0.02)$.

However in our study, mortality rate was quite high $34.5 \%$ in methylprednisolone group and in $37.8 \%$ in pentoxifylline group in 28 days period. In our study most of the patients in both group were CTP C (87.2\%) and had very high DF score. Most of the patients visited hospital in late phase. This finding suggest that the transplantation is probable other modality of treatment that remain for the CTP C patients with high DF score at presentation.
This is small study and required larger scale study to support this finding. Limitation of our study were liver biopsy not performed before starting the treatment and study period being quite short (28 days).

\section{CONCLUSIONS}

Alcohol is most common beverages which are abused worldwide. Alcoholic hepatitis is common manifestation of alcoholic liver diseases. Short term (28 days) mortality is quite high in our population and most of these patients presented late with high DF. Most effective way to decrease the incidence of $\mathrm{AH}$ is to avoid alcohol drinking and early diagnosis and treatment.

\section{Conflict of Interest:}

None

\section{REFERENCES}

1. Crabb DW, Bataller R, Chalasani NP, Kamath PS, Lucey M, Mathurin P, McClain C, McCullough A, Mitchell MC, Morgan TR, Nagy L. Standard definitions and common data elements for clinical trials in patients with alcoholic hepatitis: recommendation from the NIAAA Alcoholic Hepatitis Consortia. Gastroenterology. 2016 Apr 1;150(4):785-90.

2. Sandahl TD, Jepsen P, Thomsen KL, et al. Incidence and mortality of alcoholic hepatitis in Denmark 1999-2008: a nationwide population based cohort study. J Hepatol 2011;54:760-764.

3. Forrest EH, Evans CD, Stewart S, et al. Analysis of factors predictive of mortality in alcoholic hepatitis and derivation and validation of the Glasgow alcoholic hepatitis score. Gut 2005; 54:1174-1179.

4. Nguyen-Khac E, Thevenot T, Piquet MA, et al. Glucocorticoids plus N-acetylcysteine in severe alcoholic hepatitis. N Engl J Med 2011;365:1781-9.

5. Rahimi E, Pan JJ. Prognostic models for alcoholic hepatitis. Biomarker research. 2015 Dec;3(1):20.

6. Simon D, Galambos JT. A randomized controlled study of peripheral parenteral nutrition in moderate and severe alcoholic hepatitis. J Hepatol 1988; 7:200.

7. Cabré E, et al. Short- and long-term outcome of severe alcohol-induced hepatitis treated with steroids or enteral nutrition: a multicenter randomized trial. Hepatology. 2000; 32:36-42.

8. Thursz MR, Richardson P, Allison M, Austin A, Bowers M, Day CP, et al. Prednisolone or pentoxifylline for alcoholic hepatitis. N Engl J Med 2015;372:1619-1628.

9. Singh S, Murad MH, Chandar AK, Bongiorno CM, Singal AK, Atkinson SR, et al. Comparative effectiveness of pharmacological interventions for severe alcoholic hepatitis: a systematic review and network metaanalysis. Gastroenterology 2015;149:958-970, e912.

10. Mathurin P, Abdelnour M, Ramond MJ, et al. Early change in bilirubin levels is an important prognostic factor in severe alcoholic hepatitis treated with prednisolone. Hepatology 2003;38:1363-9.

11. Gholam PM . Prognosis and prognostic scoring models for alcoholic liver disease and acute alcoholic hepatitis . Clin Liver Dis $2016 ; 20: 491-7$.

12. Dunn W, Jamil LH, Brown LS et al. MELD accurately predicts mortality in patients with alcoholic hepatitis . Hepatology $2005 ; 41: 353-8$.

13. Louvet A, Naveau S, Abdelnour M et al. Th e Lille model: a new tool for therapeutic strategy in patients with severe alcoholic hepatitis treated with steroids. Hepatology $2007 ; 45: 1348-54$.

14. Mookerjee RP, Malaki M, Davies NA, Hodges SJ, Dalton RN, Turner C, et al. Increasing dimethylarginine levels are associated with adverse clinical outcome in severe alcoholic hepatitis. Hepatology 2007;45:62-71.

15. Carithers RL, Herlong HF, Diehl AM, Shaw EW, Combes B, Fallon HJ, Maddrey WC. Methylprednisolone therapy in patients with severe alcoholic hepatitis: a randomized multicenter trial. Annals of internal medicine. 1989 May 1;110(9):685-90. 